\title{
Article \\ Neural Network-Based Calculator for Rat Glomerular Filtration Rate
}

\author{
Óscar J. Pellicer-Valero 1,+(D), Giampiero A. Massaro $2,3,4,5,6,+\mathbb{D}$, Alfredo G. Casanova $2,3,4,5,6 \mathbb{D}$, \\ María Paniagua-Sancho $2,3,4,5,6$, Isabel Fuentes-Calvo ${ }^{2,3,5,6}$, Mykola Harvat ${ }^{1}$, José D. Martín-Guerrero 1,7, $\ddagger$, \\ Carlos Martínez-Salgado $2,3,5,6,7, \mp$ iD and Francisco J. López-Hernández $2,3,4,5,6,7,8, *, \ddagger$ (D)
}

Citation: Pellicer-Valero, Ó.J.; Massaro, G.A.; Casanova, A.G.; Paniagua-Sancho, M.; Fuentes-Calvo, I.; Harvat, M.; Martín-Guerrero, J.D.; Martínez-Salgado, C.; López-Hernández, F.J. Neural Network-Based Calculator for Rat Glomerular Filtration Rate. Biomedicines 2022, 10, 610. https:/ / doi.org/10.3390/biomedicines 10030610

Academic Editor: Jun Lu

Received: 6 February 2022

Accepted: 3 March 2022

Published: 5 March 2022

Publisher's Note: MDPI stays neutral with regard to jurisdictional claims in published maps and institutional affiliations.

Copyright: (c) 2022 by the authors Licensee MDPI, Basel, Switzerland. This article is an open access article distributed under the terms and conditions of the Creative Commons Attribution (CC BY) license (https:// creativecommons.org/licenses/by/ $4.0 /)$.
1 Intelligent Data Analysis Laboratory (IDAL), Department Electronic Engineering, School of Engineering (ETSE-UV), Universitat de València, 46100 Valencia, Spain; oscar.pellicer@uv.es (Ó.J.P.-V.); mykola.harvat@uv.es (M.H.); jose.d.martin@uv.es (J.D.M.-G.)

2 Institute of Biomedical Research of Salamanca, 37007 Salamanca, Spain; giampieroandrea.massaro@usal.es (G.A.M.); alfredogcp@usal.es (A.G.C.); meripani@usal.es (M.P.-S.); ifc@usal.es (I.F.-C.); carlosms@usal.es (C.M.-S.)

3 Departmento de Fisiología y Farmacología, Universidad de Salamanca, 37007 Salamanca, Spain

4 Fundación Instituto de Estudios de Ciencias de la Salud de Castilla y León, 42002 Soria, Spain

5 Group of Translational Research on Renal and Cardiovascular Diseases (TRECARD), 37007 Salamanca, Spain

6 National Network for Kidney Research REDINREN, RD016/0009/0025, Instituto de Salud Carlos III, 28029 Madrid, Spain

7 Disease and Theranostic Modelling Consortium (DisMOD), 37007 Salamanca, Spain

8 Group of Biomedical Research on Critical Care (BioCritic), 47003 Valladolid, Spain

* Correspondence: flopezher@usal.es

+ These authors contributed equally to this work.

$\ddagger$ These authors contributed equally to this work.

\begin{abstract}
Glomerular filtration is a pivotal process of renal physiology, and its alterations are a central pathological event in acute kidney injury and chronic kidney disease. Creatinine clearance $(\mathrm{ClCr})$, a standard method for glomerular filtration rate (GFR) measurement, requires a long and tedious procedure of timed (usually $24 \mathrm{~h}$ ) urine collection. We have developed a neural network $(\mathrm{NN})$-based calculator of rat $\mathrm{ClCr}$ from plasma creatinine $(\mathrm{pCr})$ and body weight. For this purpose, matched $\mathrm{pCr}$, weight, and $\mathrm{ClCr}$ trios from our historical records on male Wistar rats were used. When evaluated on the training (1165 trios), validation (389), and test sets (660), the model committed an average prediction error of $0.196,0.178$, and $0.203 \mathrm{~mL} / \mathrm{min}$ and had a correlation coefficient of $0.863,0.902$, and 0.856 , respectively. More importantly, for all datasets, the NN seemed especially effective at comparing $\mathrm{ClCr}$ among groups within individual experiments, providing results that were often more congruent than those measured experimentally. ACLARA, a friendly interface for this calculator, has been made publicly available to ease and expedite experimental procedures and to enhance animal welfare in alignment with the 3Rs principles by avoiding unnecessary stressing metabolic caging for individual urine collection.
\end{abstract}

Keywords: rat glomerular filtration rate; creatinine clearance; calculator; neural network; machine learning

\section{Introduction}

Alterations in GFR are a hallmark of many renal ailments, including acute kidney injury (AKI) [1] and chronic kidney disease (CKD) [2,3], and a gold standard diagnostic parameter [4-6]. Methods to measure GFR are based on the clearance of specific probe molecules from the blood. These must be cleared solely by renal excretion, not metabolized, freely filtered at the glomerulus, or secreted or reabsorbed by the tubule, and must not interfere with the GFR [7,8]. Different exogenous molecules with these characteristics have 
been used [8-10], but the clearance of inulin (CIIn), a plant fructan oligosaccharide, is still the gold standard GFR measurement method [7,11-13].

These clearance methods are overtly inconvenient for routine clinical practice $[14,15]$ due to the time-consuming and costly procedures, such as the need for exogenous probe administration, timed urine and blood collection and analysis, and the involvement of radioactivity [9]. For these reasons, GFR measurement turned to endogenous probes [16], the most used of which is creatinine $(\mathrm{Cr})$, a metabolite of phosphocreatine and a waste product of muscle activity. Creatinine clearance $(\mathrm{ClCr})$ is not the ideal method for GFR measurement either, because $\mathrm{Cr}$ is also secreted in the tubule and, in some circumstances, can also be cleared from the organism with the feces [17]. In fact, $10-40 \%$ of the creatinine found in the urine is secreted in normal individuals [18], which leads to an overestimation of the GFR when measured as $\mathrm{ClCr}$, compared to ClIn [19]. Yet endogenous $\mathrm{ClCr}$, introduced in 1937 [20], avoids probe administration and the use of radioactivity because $\mathrm{Cr}$ can be measured with a colorimetric reaction known as Jaffe's method [7,21,22], or with enzymatic assays [23]. Its reduced complexity, cost, and risk rapidly led to the adoption of $\mathrm{ClCr}$ [7], which still requires timed urine sampling (usually 24-h collection).

Serum creatinine concentration ( $\mathrm{sCr}$ ) is a gross and inverse reflection of $\mathrm{ClCr}$ and, thus, of the GFR because it is produced in the muscle at a more or less constant average daily rate. Variations in $\mathrm{sCr}$ are therefore mostly derived from changes in $\mathrm{ClCr}$. Accordingly, with all its limitations [24,25], sCr was progressively adopted as the election proxy to estimate GFR, especially after methods for automatic analysis were developed by the 1960s [21,25]. Still, $\mathrm{sCr}$ is dependent on muscle mass, race, and other factors; thus, algorithms have been developed to estimate GFR from sCr and personal and body habitus data [9]. In 1976, the Schwartz formula (adjusted by height) [26] and the Cockroft-Gault equation (adjusted by age and weight) [27] were published. Over 25 formulas have been developed thereafter [28], most notably including the Modification of Diet in Renal Disease equation (MDRD) [29] and the Chronic Kidney Disease Epidemiology Collaboration (CKD-EPI) group equation [30], both adjusted by age, sex, and ethnicity. Due to its reasonably high accuracy and practicality, estimated GFR has substituted measured GFR (i.e., clearance methods) in most clinical situations [4]. Recently, a novel approach has been proposed to efficiently estimate GFR from $\mathrm{sCr}$ through a numerical algorithm based on bivariate numerical modeling [31].

Similarly, in experimental research with animals, ClIn $[32,33]$ is the preferred method for GFR assessment, but endogenous $\mathrm{ClCr}[34,35]$ is most widely used due to reduced complexity. Still, individual, laborious 24-hour urine collection is necessary. A transcutaneous exogenous fluorescence-labeled sinistrin clearance (CISin) method has been developed which avoids individual housing and allows longitudinal studies with minimal invasiveness [36]. However, ClSin is also laborious and technically more demanding than traditional clearance techniques. Due to simplicity, $\mathrm{sCr}$ is again the most frequent surrogate for GFR.

In this study we developed and experimentally validated a neural network-based calculator of $\mathrm{ClCr}$ for rats from a single analyte (i.e., $\mathrm{sCr}$ ) and body weight data, useful in both healthy and disease conditions. This calculator may be used in many acute and chronic pathophysiological circumstances to prevent long, tedious, and costly procedures and avoid the stressful conditions for experimental animals associated with $\mathrm{ClCr}$ measurement.

\section{Materials and Methods}

\subsection{Data Mining and Database Generation}

To train the model, 1554 data trios of measured $\mathrm{ClCr}(\mathrm{mClCr}), \mathrm{pCr}$, and body weight were collected from the historical records of our laboratory. These datasets came from published and unpublished experiments with AKI and CKD models (and their healthy, normal controls) (Figure 1 and Table 1). A random subset with $75 \%$ of the data trios (1165) was used for model training (i.e., the training set), and the remaining $25 \%$ (389) was used for validation (i.e., the validation set), which was utilized for making design decisions (e.g., model selection, preprocessing pipeline design, etc.). Once the final model was obtained, it 
was field-tested against a third dataset with 660 data trios (i.e., the test set) from rats and experiments different from those used for training and validation. In all cases, the data arose from male Wistar rats, with no exclusion criteria. $\mathrm{ClCr}$ had been measured as we previously described [37,38]. Briefly, $\mathrm{ClCr}$ had been determined with the following formula: $\mathrm{ClCr}=\mathrm{UF} 24 \mathrm{~h} \times \mathrm{uCr} / \mathrm{pCr}$, where UF24h is the volume of urine collected in $24 \mathrm{~h}, \mathrm{pCr}$ is the plasma concentration of creatinine, and $\mathrm{uCr}$ is the urinary concentration of creatinine. For individual urine collection, rats had been allocated in metabolic cages and allowed free access to water and regular chow. Blood samples had been collected from a small incision in the tail and immediately centrifuged to obtain the plasma, which was frozen at $-80{ }^{\circ} \mathrm{C}$ until use. Both $\mathrm{pCr}$ and $\mathrm{uCr}$ had been measured with a commercial colorimetric assay based on Jaffe's method (Quantichrom Creatinine Assay Kits, BioAssay Systems, Hayward, CA, USA) following the manufacturer's instructions. A database was generated with rat identifiers, experimental model, $\mathrm{ClCr}, \mathrm{pCr}$, and body weight values.

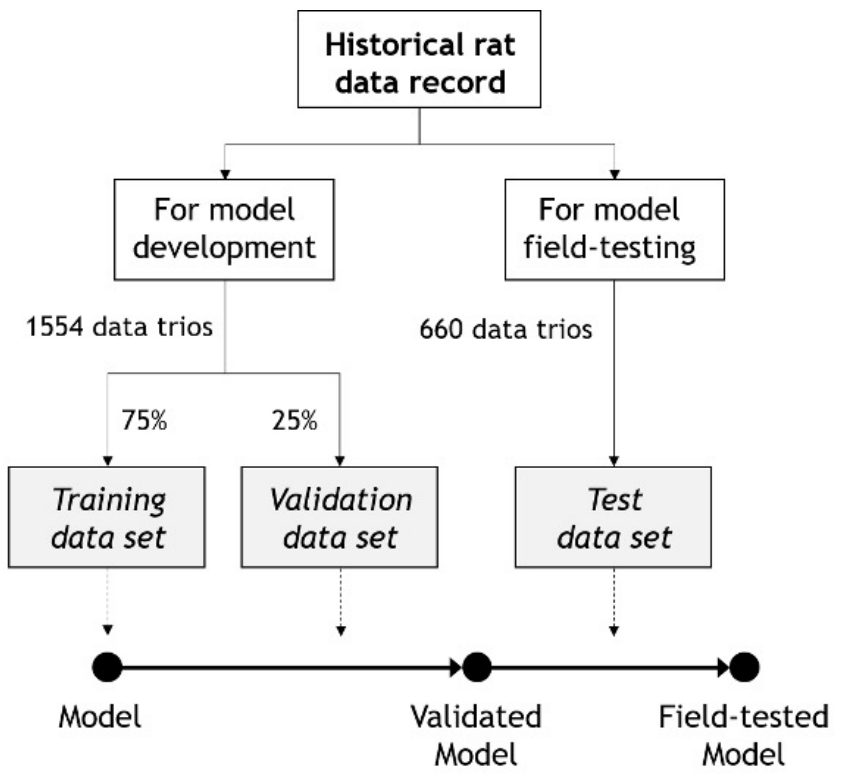

Figure 1. Summary of the datasets used for model development and field testing. Data trios were composed of matched $\mathrm{pCr}, \mathrm{ClCr}$, and body weight data.

Table 1. Characteristics of the datasets used for model development and field testing. CTRL, control. T-AKI, toxic acute kidney injury. I/R, ischemia/reperfusion. $M$, male. RMR, 5/6 renal mass reduction. PRD, predisposed-to-AKI (as in [39-42]).

\begin{tabular}{ccccc}
\hline Model & $\begin{array}{c}\mathbf{n} \\
\text { (Rats) }\end{array}$ & $\begin{array}{c}\text { Age } \\
\text { at Start } \\
\text { (Weeks) }\end{array}$ & $\begin{array}{c}\text { Duration } \\
\text { (Weeks) }\end{array}$ & $\begin{array}{c}\text { nata Trios) } \\
\text { (Dodel Development (Training + Validation Datasets) }\end{array}$ \\
\hline CTRL & 9 & 8 & 1 & 51 \\
T-AKI & 34 & 8 & $2-28$ & 194 \\
& 109 & 8 & 1 & 583 \\
I/R & 19 & 8 & $2-8$ & 38 \\
RMR & 64 & 8 & $2-28$ & 63 \\
& 15 & 8 & $2-8$ & 184 \\
\hline CTRL & 35 & 8 & $2-28$ & 59 \\
& & Test Dataset & 1 & 21 \\
PRD & 13 & 8 & 5 & 279 \\
T-AKI & 2 & 8 & 7 & 38 \\
& 3 & 8 & 1 & 49 \\
& 61 & 8 & 5 & 204 \\
\hline
\end{tabular}




\subsection{Algorithm Description}

Linear regression (LR), random forest (RF), and feedforward neural network (FFNN) $[43,44]$ models were evaluated comparatively. Among them, LR is the simplest algorithm as it can only learn independent linear relationships between each input feature and the output. Conversely, RF is a non-linear model able to capture complex relationships among input and output features as it uses the compound prediction from many (e.g., 100) decision trees to perform regression. Finally, FFNNs are connectionist models that allow for fitting non-linear relationships by stacking many LR units (i.e., neurons) with non-linear activation functions in between, similar to how simple biological neurons combine to form a complex net: the brain. For the LR and RF models, the Scikit-learn library (version 0.24.1) [45] was employed, while for the FFNN, TensorFlow (version 2.1.0) [46] was used.

\subsection{Data Preprocessing}

The relationship between $\mathrm{pCr}$ and $\mathrm{mClCr}$ was linearized by logarithmic transformation (Equation (1) and Supplementary Figure S1) to help the ML algorithms better capture the relationship. The input features ( $\mathrm{pCr}$ and weight) were then standardized by Equation (2), which centers the data distribution around zero and scales it to unit variance. Standardization is required by LR, and it is helpful for training other ML models too since it equalizes the relative importance of the features.

$$
\begin{aligned}
& f(x)=\log \left(\frac{1}{x}\right) \\
& \widetilde{x}=\frac{x-\operatorname{mean}(x)}{\operatorname{std}(x)}
\end{aligned}
$$

\subsection{Neural Network Training, Validation, and Testing}

For FFNN training, the parameters, i.e., weights, minimizing the model's prediction error, i.e., the mean squared error (MSE, Equation (3)), between $\mathrm{eClCr}$ and $\mathrm{mClCr}$ in the training set were found by the stochastic gradient descent (SGD) [47] iterative optimizer.

$$
\operatorname{MSE}\left(e C l_{C r}, m C l_{C r}\right)=\operatorname{mean}\left(\left(e l_{C r}-m C l_{C r}\right)^{2}\right)
$$

Model performance was mainly assessed with the mean absolute error (MAE) and the Pearson product-moment correlation coefficient (Equations (4) and (5), respectively), although p10 and p30 metrics were also included for completeness. The MAE represents the absolute error (in $\mathrm{mL} / \mathrm{min}$ ) that is committed on average in every prediction. The correlation coefficient measures the strength of the relationship between $\mathrm{mCl} C \mathrm{Cr}$ and $e \mathrm{Cl} \_\mathrm{Cr}$ with a value between 0 and 1, where 1 stands for a perfect prediction performance (i.e., $e \mathrm{ClCr}=m \mathrm{ClC}$ for all data trios). Finally, p10 and p30 represent the fraction of predictions $\left(e C l \_C r\right)$ that fall within $10 \%$ and $30 \%$ of the $m C l \_C r$, respectively.

$$
\begin{aligned}
& \operatorname{MAE}\left(e \mathrm{Cl}_{C r}, m C l_{C r}\right)=\operatorname{mean}\left(\left|e C l_{C r}-m C l_{C r}\right|\right) \\
& \operatorname{corr}\left(e C l_{C r}, m C l_{C r}\right)=\frac{\operatorname{cov}\left(e C l_{C r}, m C l_{C r}\right)}{\operatorname{std}\left(m C l_{C r}\right) \cdot \operatorname{std}\left(e C l_{C r}\right)}
\end{aligned}
$$

To avoid potential overfitting, all engineering decisions (FFNN architecture, number of layers, neurons per layer, activation functions, etc.) were made based on the validation MAE and correlation coefficient.

Once the model (NN architecture + weights) was finalized, it was challenged with the test set as a proxy for field performance. 


\section{Results}

\subsection{Model Development, Evaluation, and Validation}

With the objective of developing a machine learning (ML) model (i.e., a calculator) to estimate rat $\mathrm{ClCr}$ (i.e., estimated $\mathrm{ClCr}$; eClCr) from plasma creatinine concentration $(\mathrm{pCr}$ ) and body weight, three ML algorithms were assessed and compared: LR, RF, and FFNN. Table 2 shows the performance metrics for the three models after training and tuning them. The FFNN outperformed the LR and RF models on the validation set and was therefore selected for further testing. Values of $\mathrm{eClCr}$ were compared to the measured (i.e., experimental) values of $\mathrm{ClCr}(\mathrm{mClCr})$.

Table 2. Performance metrics for the three considered models in the training and validation sets. LR, linear regression. RF, random forest. FFNN, feed forward neural network. MAE, mean average error. Correlation, Pearson product-moment correlation coefficient. P10/P30, fraction of predictions with an error within the $10 \% / 30 \%$ threshold. Best results for each set are highlighted in bold.

\begin{tabular}{|c|c|c|c|c|c|c|c|c|c|}
\hline & \multicolumn{3}{|c|}{ Training Set } & \multicolumn{3}{|c|}{ Validation Set } & \multicolumn{3}{|c|}{ Test Set } \\
\hline & LR & RF & FFNN & LR & RF & FFNN & LR & RF & FFNN \\
\hline MAE & 0.2210 & 0.1801 & 0.1956 & 0.1975 & 0.1894 & 0.1780 & 0.2515 & 0.2257 & 0.2035 \\
\hline Correlation & 0.8402 & 0.8869 & 0.8632 & 0.8732 & 0.8871 & 0.9028 & 0.7922 & 0.7450 & 0.8563 \\
\hline P10 & 0.2635 & 0.3322 & 0.3099 & 0.2699 & 0.2828 & 0.2879 & 0.2530 & 0.2803 & 0.3136 \\
\hline P30 & 0.6901 & 0.7751 & 0.7391 & 0.7044 & 0.7189 & 0.7532 & 0.6394 & 0.6727 & 0.6955 \\
\hline
\end{tabular}

The optimal FFNN architecture was found to have three intermediate (hidden) layers with 30, 20, and 10 neurons respectively, with the hyperbolic tangent as an activation function and using the Adam SGD optimizer with a learning rate of 0.001 and a batch size of 400 with MSE as the loss function. With the aid of the early stopping technique, training was stopped after 200 SGD iterations without improvement of the validation loss. The characteristics of the final FFNN architecture are shown in Supplementary Figure S2 and a decision surface map for the FFNN is shown in Supplementary Figure S3. Agreement between $\mathrm{eClCr}$ and $\mathrm{mClCr}$ was also studied in the test set by means of a Bland-Altman plot (Supplementary Figure S4).

In addition to analyzing the global performance of the model, the results of eClCr were also compared with their corresponding $\mathrm{mClCr}$ values in the context of individual experiments. Figure 2 shows the $\mathrm{mClCr}$ and $\mathrm{eClCr}$ for four representative experiments (from the training and validation datasets). In general terms, the information provided by measured $\mathrm{ClCr}(\mathrm{mClCr})$ and $\mathrm{eClCr}$ is almost identical. In particular, in those cases in which $\mathrm{mClCr}$ and eClCr are less similar (e.g., groups D2.1 and D2.5 in Figure 2), eClCr is consistently more congruent within its context. Just as an example, the group D2.1 is a control, and groups D2.2 to D2.5 refer to animals treated with cisplatin in several conditions. There is no reason for $\mathrm{mClCr}$ to increase in group D2.5 on day 5. On the contrary, following a similar pattern to the other AKI groups, eClCr shows a more logical result for group D2.5 and overall.

EXPERIMENT \#D1

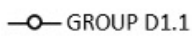

$\rightarrow$ GROUP D1.2

- GROUP D1.3

$\rightarrow$ GROUP D1.4

$\triangle \backsim$ GROUP D1.5
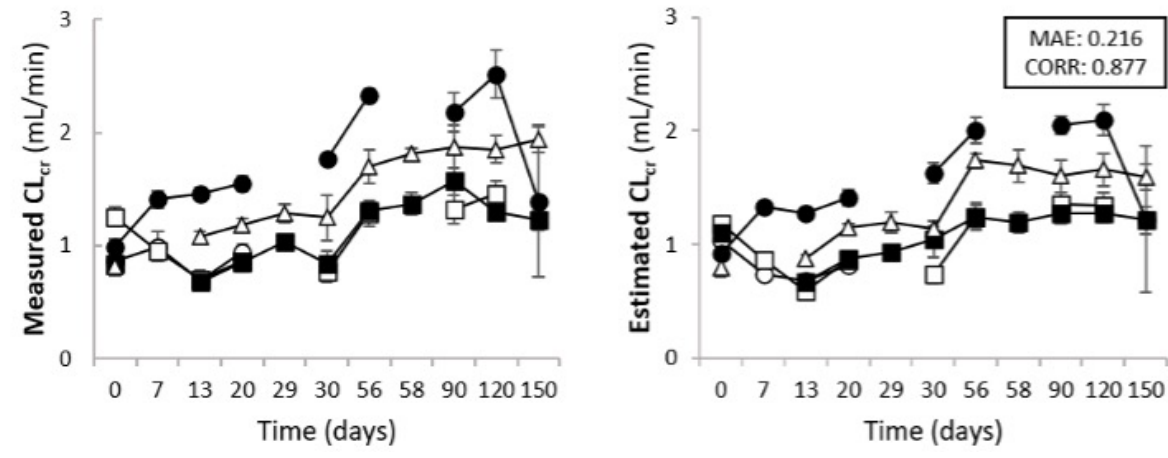

Figure 2. Cont. 
EXPERIMENT \#D2

-O-GROUP D2.1

$\rightarrow$ G-GROUP D2.2

$\rightarrow$ GROUP D2.3

- GROUP D2.4

$\triangle-$ GROUP D2.5

EXPERIMENT \#D3

$\multimap-$ GROUP D3.1.

$\rightarrow-G R O U P$ D3.2.

$\multimap$ GROUP D3.3.

$\rightarrow$-GROUP D3.4.

-O-GROUP D4.1.

$\rightarrow$-GROUP D4.2.

$\multimap$ GROUP D4.3.

- GROUP D4.4.

$\rightarrow-$ GROUP D 4.5 .
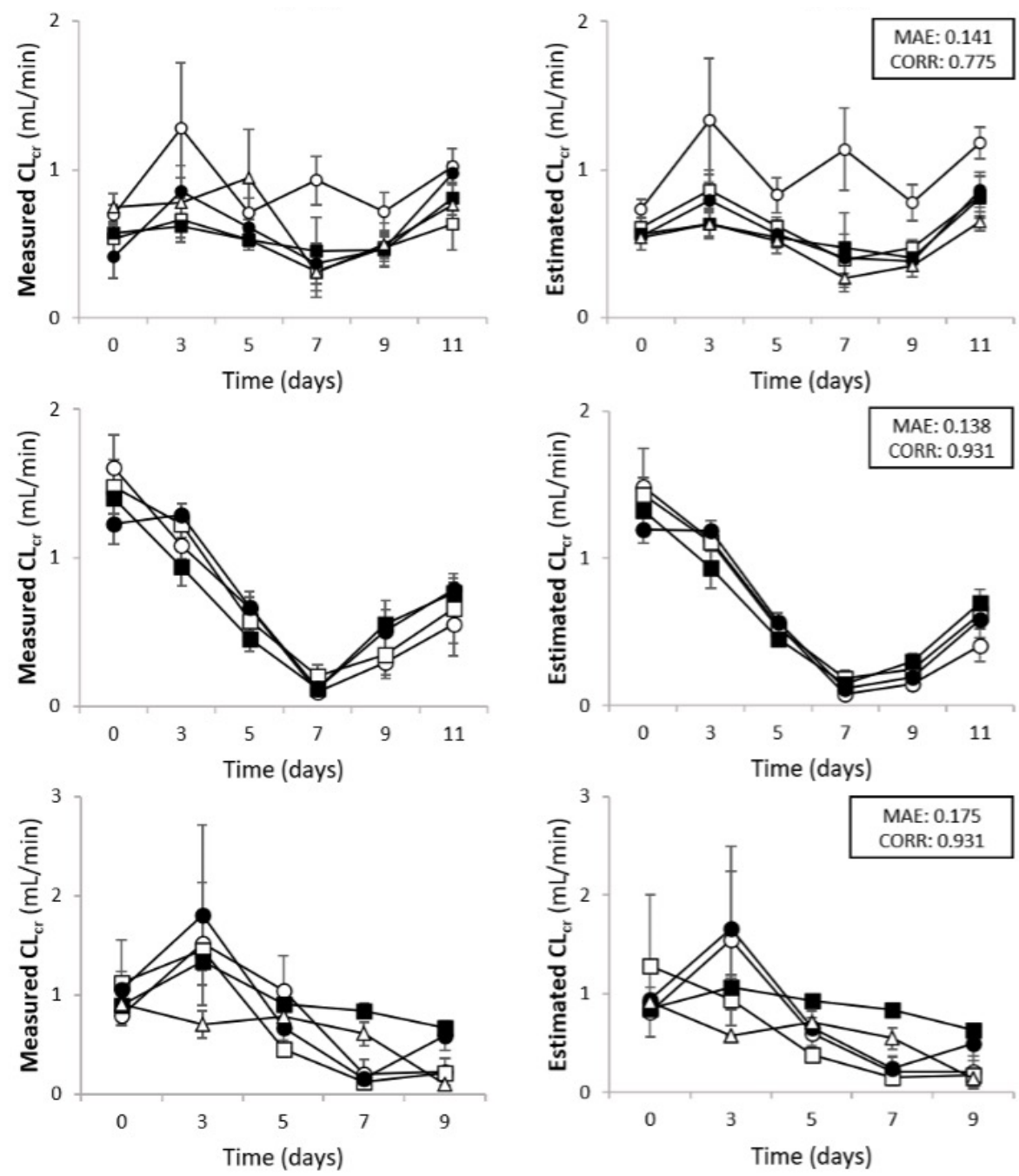

Figure 2. Comparison of the measured $\mathrm{ClCr}$ and estimated $\mathrm{ClCr}$, corresponding to four exemplifying actual experiments using data from the training and validation sets. $\mathrm{ClCr}$, creatinine clearance. CORR, Pearson product-moment correlation coefficient. MAE, mean average error.

\subsection{Model Field Testing}

As shown in Table 2, the MAE and Pearson product-moment correlation coefficient for the test set were 0.2035 and 0.8564 , respectively, which are similar to those obtained in the training and validation sets, hence proving that the model is not overfitted and can generalize to unseen data. Furthermore, a paired $t$-test was employed to compare the mean of the absolute error committed by the NN and the LR models in the test set; the NN was found to be superior with very high statistical significance $(p<0.001$, using SciPy library [48], version 1.5.2). As above, eClCr predictions were compared with their corresponding $\mathrm{mClCr}$ values in the context of specific experiments. Figure 3 shows the $\mathrm{mClCr}$ and $\mathrm{eClCr}$ for four illustrative experiments (from the test data). There is a good match at the whole experiment level between $\mathrm{mClCr}$ and eClCr. Again, eClCr is more congruent than $\mathrm{mClCr}$. As exemplifying tokens, the $\mathrm{mClCr}$ in group $\mathrm{T} 1.5$ by day 2 and group T1.1 by day 8 (a control) shows illogical increments that are normalized or softened by the corresponding eClCr. In experiment $\mathrm{T} 2$, the open circles correspond to the control group and the filled circles to a highly nephrotoxic, one-dose treatment. As expected, by day 4 , there is a notorious drop in the GFR, which is progressively restored afterwards. The gradual recovery described by eClCr contrasts with the more erratic trajectory depicted by $\mathrm{mClCr}$. 

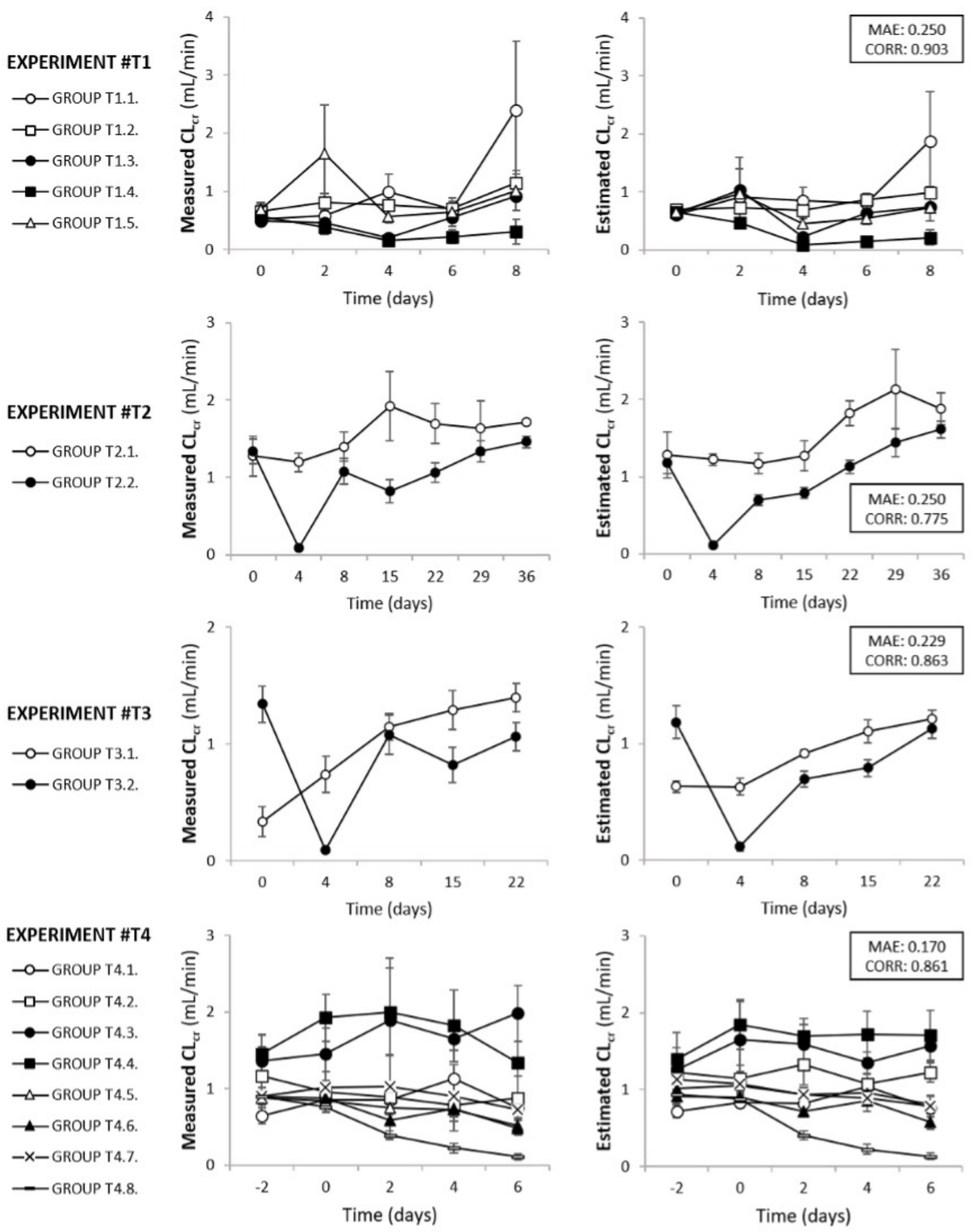

Figure 3. Comparison of the measured $\mathrm{ClCr}$ and estimated $\mathrm{ClCr}$, corresponding to four exemplifying actual experiments, using data from the test set. $\mathrm{ClCr}$, creatinine clearance. CORR, Pearson productmoment correlation coefficient. MAE, mean average error.

The influence of weight and $\mathrm{mClCr}$ on the distribution of the prediction error $(\mathrm{eClCr}-$ $\mathrm{mClCr}$ ) in the whole test set is shown in Figure 4. The variance of the prediction error grows (top panel) with the weight, probably as a result of an increasingly lower amount of data for higher weights. The medians suggest that the model may underestimate the $\mathrm{ClCr}$ for high weights. In contrast, the variance of the prediction error seems to be similar for all $\mathrm{ClCr}$ values (bottom panel). Yet the medians show that the model tends to slightly overestimate $\mathrm{ClCr}$ for small to medium $\mathrm{mClCr}$ values (below $0.683 \mathrm{~mL} / \mathrm{min}$ ) and to slightly underestimate $\mathrm{ClCr}$ for medium to larger $\mathrm{mClCr}$ values (above $1.138 \mathrm{~mL} / \mathrm{min}$ ). 

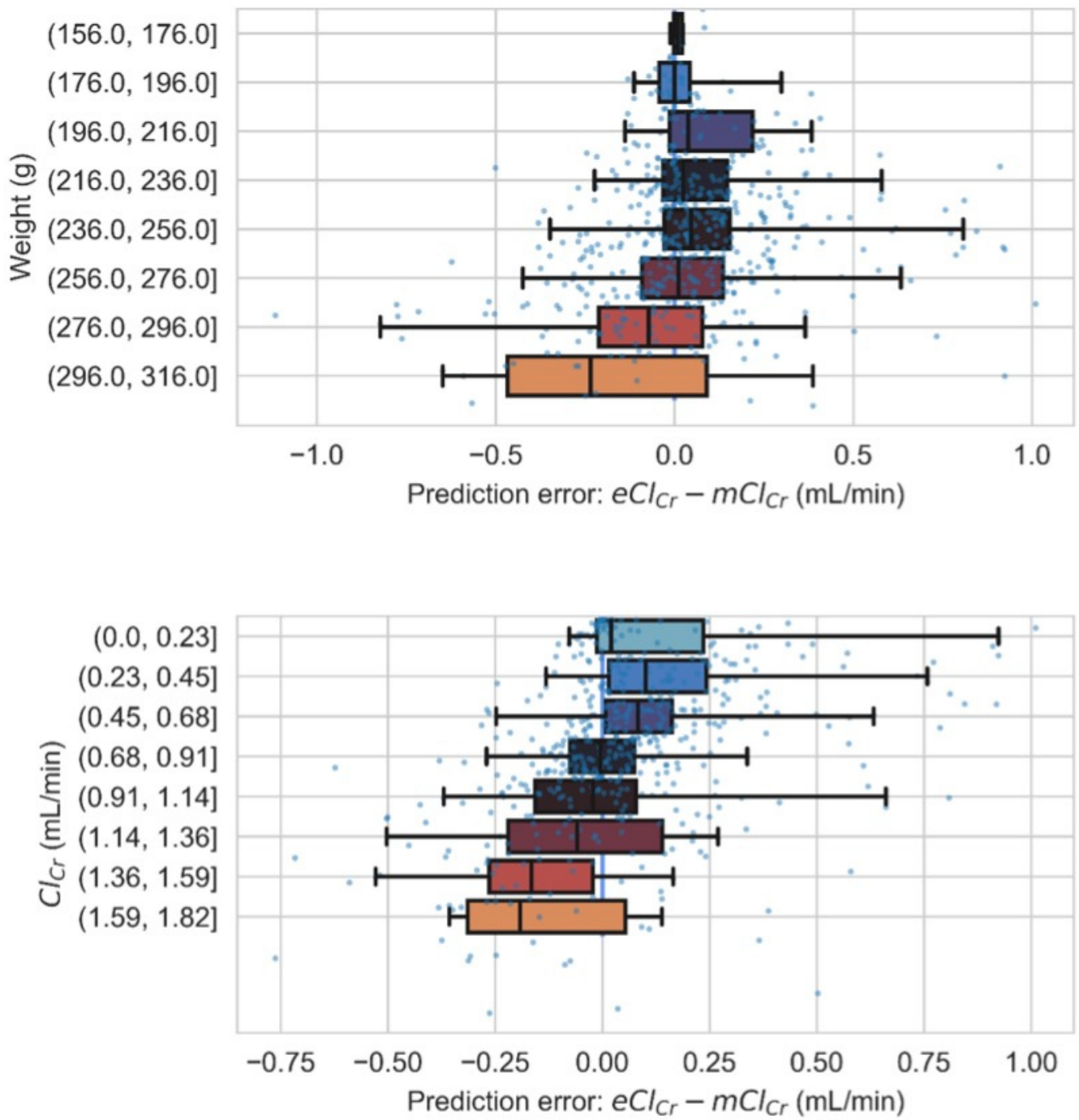

Figure 4. Prediction error analysis. Prediction error box plots according to the rat's body weight range (top) and the $\mathrm{mClCr}$ range (bottom). Error bars represent the 2.5 th and 97.5 th percentiles. A scatter plot of prediction errors is also shown superimposed.

\section{Discussion}

Our study reveals that an FFNN fed with $\mathrm{pCr}$ and weight values as inputs predicts $\mathrm{ClCr}$ (i.e., eClCr) with reasonably high accuracy, regardless of additional variables such as age, comorbidities, or treatment. This calculator may be useful for the estimation of $\mathrm{ClCr}$ in those laboratories where metabolic cages are not available, or when avoidance of experimental complexity and resource optimization are sought.

When evaluated with the training, validation, and test datasets, the model showed a relatively low average error. Furthermore, the model proved especially useful for evaluating experiments on the whole. In fact, the behavior and evolution of eClCr for each group was, in general, more homogeneous and congruent than the behavior of $\mathrm{mClCr}$, while the relations among experimental groups resulting from $\mathrm{eClCr}$ remained extremely similar to those obtained with $\mathrm{mClCr}$. Our interpretation of this data-equalization effect is that, as opposed to $\mathrm{mClCr}, \mathrm{eClCr}$ obviates the error introduced by urine collection in metabolic cages, which is unavoidably translated to $\mathrm{uCr}$ estimation and, in turn, to $\mathrm{mClCr}$, and thus sometimes yields unexplainable and illogical fluctuations (as argued in the Results section). This advantage adds to the procedural simplicity of eClCr compared to $\mathrm{mClCr}$. Accordingly, this calculator is not aimed at being a method for the precise determination of $\mathrm{ClCr}$ but a practical tool providing rapid, easy, and sufficiently accurate results for many 
purposes of basic research with rats, in the same way that human GFR-estimating formulas are useful in the clinical setting. In our view, this calculator is particularly appropriate for those experiments in which it is not the exact individual value of $\mathrm{ClCr}$ that is required but rather its comparison among experimental groups or time points. In addition to facilitating preclinical research, $\mathrm{eClCr}$ also provides an alternative to $\mathrm{mClCr}$ that improves experimental animal welfare. Individual confinement in metabolic cages $[37,38]$ required for urine collection is known to stress social animals such as rats [49]. Hence, this method is tightly aligned with the 3Rs principles (replacement, reduction, and refinement) concerning animal research [50].

In rats, $\mathrm{pCr}$ is mostly constant throughout their lifespan. However, the GFR (and thus $\mathrm{ClCr}$ ) increases within an initial age range, after which it largely stabilizes [51]. Therefore, age should be taken into account when modeling the $\mathrm{ClCr}-\mathrm{pCr}$ relationship. Yet because rat age might not be precisely known by the researcher in some cases, we used weight as a surrogate and found that its inclusion significantly improved model performance (even more than age itself). Furthermore, we contend that weight recapitulates other potential sources of disparity related to body habitus. Ethnicity and sex were not included in the model as we did not have sufficient data from other rat strains, nor of female rats. Accordingly, this model is adjusted only for male Wistar rats. Its performance must also be tested, and the model adjusted (if and as necessary), for different rat strains and female sex.

Another important factor potentially uncoupling the relationship between $\mathrm{pCr}$ and $\mathrm{ClCr}$ is creatinine secretion. Differential affecting of creatinine secretion by different experimental conditions might increase variability and hamper model performance. However, the model was generated on heterogeneous data from etiologically diverse disease models, and there were no deviation tendencies to specific characteristics. This implies that creatinine secretion is not significantly modified by the treatments or conditions included and that the observed data variability mainly reflects experimental variability derived from sample collection and analytic error.

\section{Conclusions}

The proposed model is a reasonably accurate surrogate for $\mathrm{mClCr}$, solves some of its faults, and even provides more congruent results. eGFR in humans is known to be less accurate in specific populations and circumstances, such as individuals with abnormal muscle mass or body surface area; during changes in metabolism and pregnancy; and when GFR is rapidly changing, such as during growth, AKI, or high-protein ingestion [16,52]. In contrast, laboratory rats present a lower phenotypic heterogeneity as compared to humans, so a higher accuracy is therefore expected. Lastly, we have released ACLARA, a web-based, openly available eClCr calculator (http:/ /idal.uv.es/aclara, accessed on 5 February 2022), to facilitate its use and encourage further research on the topic.

Supplementary Materials: The following supporting information can be downloaded at: https:/ / www.mdpi.com/article/10.3390/biomedicines10030610/s1, Figure S1: Data transformation. Figure S2: Graphical description of the FFNN architecture. Figure S3: FFNN decision surface map. Figure S4: Bland-Altman plot of agreement between $\mathrm{eClCr}$ and $\mathrm{mClCr}$ in the test set.

Author Contributions: G.A.M., A.G.C., M.P.-S. and I.F.-C. carried out data collection and databases. Ó.J.P.-V., M.H. and J.D.M.-G. developed the mathematical model. A.G.C., Ó.J.P.-V., G.A.M. and M.P.-S. made the figures and tables. C.M.-S., J.D.M.-G. and F.J.L.-H. designed the study. Ó.J.P.-V., C.M.-S., J.D.M.-G. and F.J.L.-H. drafted the manuscript. All authors critically revised and approved the final text. All authors have read and agreed to the published version of the manuscript.

Funding: This research was funded by grants from the Government of Spain (Instituto de Salud Carlos III) (PI18/00996, PI21/01226, and Retic RD021/0005/0004, REDINREN), co-funded by FEDER funds, and from the Ministry of Education, Government of Castile and León (IES160P20), co-funded by FEDER funds.

Institutional Review Board Statement: Not applicable. 
Informed Consent Statement: Not applicable.

Data Availability Statement: Data will be available upon reasonable request.

Conflicts of Interest: The authors declare no conflict of interest.

\section{References}

1. Basile, D.P.; Anderson, M.D.; Sutton, T.A. Pathophysiology of acute kidney injury. Compr. Physiol. 2012, 2, 1303-1353. [CrossRef] [PubMed]

2. López-Novoa, J.M.; Martínez-Salgado, C.; Rodríguez-Peña, A.B.; Hernández, F.J.L. Common pathophysiological mechanisms of chronic kidney disease: Therapeutic perspectives. Pharmacol. Ther. 2010, 128, 61-81. [CrossRef] [PubMed]

3. López-Novoa, J.M.; Rodríguez-Peña, A.B.; Ortiz, A.; Martínez-Salgado, C.; López Hernández, F.J. Etiopathology of chronic tubular, glomerular and renovascular nephropathies: Clinical implications. J. Transl. Med. 2011, 9, 13. [CrossRef] [PubMed]

4. Murphy, D.P.; Hsu, C.Y. Estimating glomerular filtration rate: Is it good enough? and is it time to move on? Curr. Opin. Nephrol. Hypertens. 2013, 22, 310-315. [CrossRef] [PubMed]

5. Levey, A.S.; Becker, C.; Inker, L.A. Glomerular filtration rate and albuminuria for detection and staging of acute and chronic kidney disease in adults: A systematic review. JAMA J. Am. Med. Assoc. 2015, 313, 837-846. [CrossRef] [PubMed]

6. KDIGO. Clinical Practice Guideline for Acute Kidney Injury. Kidney Int. Suppl. 2012. Available online: https://kdigo.org/ guidelines/ckd-evaluation-and-management/ (accessed on 5 February 2022).

7. Diskin, C.J. Creatinine and glomerular filtration rate: Evolution of an accommodation. Ann. Clin. Biochem. 2007, 44, 16-19. [CrossRef] [PubMed]

8. Aurell, M. Accurate and feasible measurements of GFR-is the iohexol clearance the answer? Nephrol. Dial. Transplant. 1994, 9, 1222-1224. [CrossRef] [PubMed]

9. Schwartz, G.J.; Furth, S.L. Glomerular filtration rate measurement and estimation in chronic kidney disease. Pediatr. Nephrol. 2007, 22, 1839-1848. [CrossRef] [PubMed]

10. Delanaye, P.; Ebert, N.; Melsom, T.; Gaspari, F.; Mariat, C.; Cavalier, E.; Björk, J.; Christensson, A.; Nyman, U.; Porrini, E.; et al. Iohexol plasma clearance for measuring glomerular filtration rate in clinical practice and research: A review. Part 1: How to measure glomerular filtration rate with iohexol? Clin. Kidney J. 2016, 9, 682-699. [CrossRef] [PubMed]

11. Smith, H. The inulin clearance. In The Kidney; Smith, H., Ed.; Oxford University Press: New York, NY, USA, $1951 ;$ pp. 47-55.

12. Filler, G.; Yasin, A.; Medeiros, M. Methods of assessing renal function. Pediatr. Nephrol. 2014, 29, 183-192. [CrossRef] [PubMed]

13. Soveri, I.; Berg, U.B.; Björk, J.; Elinder, C.G.; Grubb, A.; Mejare, I.; Sterner, G.; Bäck, S.E. Measuring GFR: A systematic review. Am. J. Kidney Dis. 2014, 64, 411-424. [CrossRef] [PubMed]

14. Inker, L.A.; Levey, A.S.; Coresh, J. Estimated Glomerular Filtration Rate from a Panel of Filtration Markers-Hope for Increased Accuracy Beyond Measured Glomerular Filtration Rate? Adv. Chronic Kidney Dis. 2018, 25, 67-75. [CrossRef] [PubMed]

15. Prasad, N.; Barai, S.; Gambhir, S.; Parasar, D.; Ora, M.; Gupta, A.; Sharma, R. Comparison of glomerular filtration rate estimated by plasma clearance method with modification of diet in renal disease prediction equation and Gates method. Indian J. Nephrol. 2012, 22, 103-107. [CrossRef]

16. Rizk, D.V.; Meier, D.; Sandoval, R.M.; Chacana, T.; Reilly, E.S.; Seegmiller, J.C.; DeNoia, E.; Strickland, J.S.; Muldoon, J.; Molitoris, B.A. A novel method for rapid bedside measurement of GFR. J. Am. Soc. Nephrol. 2018, 29, 1609-1613. [CrossRef] [PubMed]

17. Wixom, R.L.; Davis, G.E.; Flynn, M.A.; Tsutakawa, R.T.; Hentges, D.J. Excretion of Creatine and Creatinine in Feces of Man. Proc. Soc. Exp. Biol. Med. 1979, 161, 452-457. [CrossRef] [PubMed]

18. Levey, A.S.; Perrone, R.D.; Madias, N.E. Serum creatinine and renal function. Annu. Rev. Med. 1988, 39, 465-490. [CrossRef]

19. Zhang, X.; Rule, A.D.; McCulloch, C.E.; Lieske, J.C.; Ku, E.; Hsu, C.Y. Tubular secretion of creatinine and kidney function: An observational study. BMC Nephrol. 2020, 21, 108. [CrossRef]

20. Popper, H.; Mandel, E. Filtrations and reabsorptions leitung in dernierenpathologie. Erg. Inn. Med. Kinder 1937, 53, 685-695.

21. Chasson, A.; Grady, H.; Stanley, M. Determination of creatinine by means of automatic chemical analysis. Tech. Bull. Regist Med. Technol. 1960, 30, 207-212. [CrossRef]

22. Husdan, H.; Rapoport, A. Estimation of creatinine by the Jaffe reaction. A comparison of three methods. Clin. Chem. 1968, 14, 222-238. [CrossRef]

23. Brück, K.; Jager, K.J.; Dounousi, E.; Kainz, A.; Nitsch, D.; Ärnlöv, J.; Rothenbacher, D.; Browne, G.; Capuano, V.; Ferraro, P.M.; et al. Methodology used in studies reporting chronic kidney disease prevalence: A systematic literature review. Nephrol. Dial. Transplant. 2015, 30, iv6-iv16. [CrossRef] [PubMed]

24. Delanaye, P.; Cavalier, E.; Pottel, H. Serum Creatinine: Not so Simple! Nephron 2017, 136, 302-308. [CrossRef] [PubMed]

25. Dennen, P.; Parikh, C.R. Biomarkers of acute kidney injury: Can we replace serum creatinine? Clin. Nephrol. 2007, 68, 269-278. [CrossRef] [PubMed]

26. Schwartz, G.; Haycock, G.; Edelmann, C., Jr.; Spitzer, A. A simple estimate of glomerular filtration rate in children derived from body length and plasma creatinine. Pediatrics 1976, 58, 259-263. [CrossRef]

27. Cockcroft, D.W.; Gault, M.H. Prediction of creatinine clearance from serum creatinine. Nephron 1976, 16, 31-41. [CrossRef]

28. Lamb, E.J.; Tomson, C.R.V.; Roderick, P.J. Estimating kidney function in adults using formulae. Ann. Clin. Biochem. 2005, 42, 321-345. [CrossRef] 
29. Levey, A.S.; Bosch, J.P.; Lewis, J.B.; Greene, T.; Rogers, N.; Roth, D. A more accurate method to estimate glomerular filtration rate from serum creatinine: A new prediction equation. Ann. Intern. Med. 1999, 130, 461-470. [CrossRef]

30. Levey, A.S.; Stevens, L.A.; Schmid, C.H.; Zhang, Y.; Castro, A.F.; Feldman, H.I.; Kusek, J.W.; Eggers, P.; Van Lente, F.; Greene, T.; et al. A new equation to estimate glomerular filtration rate. Ann. Intern. Med. 2009, 150, 604-612. [CrossRef]

31. Giles, S.N.; Fiori, S. Glomerular Filtration Rate Estimation by a Novel Numerical Binning-Less Isotonic Statistical Bivariate Numerical Modeling Method. Information 2019, 10, 100. [CrossRef]

32. Passos, M.T.; Nishida, S.K.; Câmara, N.O.S.; Shimizu, M.H.; Mastroianni-Kirsztajn, G. Iohexol clearance for determination of glomerular filtration rate in rats induced to acute renal failure. PLoS ONE 2015, 10, e0123753. [CrossRef]

33. Zhu, X.R.; Ge, N.; Wang, Y.; Zhai, J.L.; Liu, C. Corticosteroids significantly increase cystatin C levels in the plasma by promoting cystatin C production in rats. Ren. Fail. 2019, 41, 698-703. [CrossRef] [PubMed]

34. Zager, R.A. Exogenous Creatinine Clearance Accurately Assesses Filtration Failure in Rat Experimental Nephropathies. Am. J. Kidney Dis. 1987, 10, 427-430. [CrossRef]

35. Van Liew, J.B.; Zamlauski-Tucker, M.J.; Feld, L.G. Endogenous creatinine clearance in the rat: Strain variation. Life Sci. 1993, 53, 1015-1021. [CrossRef]

36. Ellery, S.J.; Cai, X.; Walker, D.D.; Dickinson, H.; Kett, M.M. Transcutaneous measurement of glomerular filtration rate in small rodents: Through the skin for the win? Nephrology 2015, 20, 117-123. [CrossRef]

37. Sancho-Martínez, S.M.; Blanco-Gozalo, V.; Quiros, Y.; Prieto-García, L.; Montero-Gómez, M.J.; Docherty, N.G.; Martínez-Salgado, C.; Morales, A.I.; López-Novoa, J.M.; López-Hernández, F.J. Impaired Tubular Reabsorption Is the Main Mechanism Explaining Increases in Urinary NGAL Excretion Following Acute Kidney Injury in Rats. Toxicol. Sci. 2020, 175, 75-86. [CrossRef] [PubMed]

38. Sancho-Martínez, S.M.; Sánchez-Juanes, F.; Blanco-Gozalo, V.; Fontecha-Barriuso, M.; Prieto-García, L.; Fuentes-Calvo, I.; González-Buitrago, J.M.; Morales, A.I.; Martínez-Salgado, C.; Ramos-Barron, M.A.; et al. Urinary TCP1-eta: A Cortical Damage Marker for the Pathophysiological Diagnosis and Prognosis of Acute Kidney Injury. Toxicol. Sci. 2020, 174, 3-15. [CrossRef]

39. Quiros, Y.; Ferreira, L.; Sancho-Martínez, S.M.; González-Buitrago, J.M.; López-Novoa, J.M.; López-Hernández, F.J. Subnephrotoxic doses of gentamicin predispose animals to developing acute kidney injury and to excrete ganglioside M2 activator protein. Kidney Int. 2010, 78, 1006-1015. [CrossRef]

40. Casanova, A.G.; Vicente-Vicente, L.; Hernández-Sánchez, M.T.; Prieto, M.; Rihuete, M.I.; Ramis, L.M.; del Barco, E.; Cruz, J.J.; Ortiz, A.; Cruz-González, I.; et al. Urinary transferrin pre-emptively identifies the risk of renal damage posed by subclinical tubular alterations. Biomed. Pharmacother. 2020, 121, 109684. [CrossRef]

41. Vicente-Vicente, L.; Sánchez-Juanes, F.; García-Sánchez, O.; Blanco-Gozalo, V.; Pescador, M.; Sevilla, M.A.; González-Buitrago, J.M.; López-Hernández, F.J.; López-Novoa, J.M.; Morales, A.I. Sub-nephrotoxic cisplatin sensitizes rats to acute renal failure and increases urinary excretion of fumarylacetoacetase. Toxicol. Lett. 2015, 234, 99-109. [CrossRef]

42. Casanova, A.G.; Fuentes-Calvo, I.; Hernández-Sánchez, M.T.; Quintero, M.; Toral, P.; Caballero, M.T.; Martínez-Salgado, C.; Morales, A.I.; Layton, A.T.; Eleno, N.; et al. The furosemide stress test and computational modeling identify renal damage sites associated with predisposition to acute kidney injury in rats. Transl. Res. 2020, 231, 76-91. [CrossRef]

43. Alpaydin, E. Introduction to Machine Learning, 4th ed.; MT Press: Cambridge, MA, USA, 2020.

44. Breinman, L. Random forests. Mach. Learn. 2001, 45, 5-32. [CrossRef]

45. Pedregosa, F.; Michel, V.; Grisel, O.; Blondel, M.; Prettenhofer, P.; Weiss, R.; Vanderplas, J.; Cournapeau, D.; Pedregosa, F.; Varoquaux, G.; et al. Scikit-learn: Machine Learning in Python. J. Mach. Learn. Res. 2011, 12, 2825-2830.

46. Abadi, M.; Agarwal, A.; Barham, P.; Brevdo, E.; Chen, Z.; Citro, C.; Corrado, G.S.; Davis, A.; Dean, J.; Devin, M.; et al. TensorFlow: Large-Scale Machine Learning on Heterogeneous Distributed Systems. arXiv 2016, arXiv:1603.04467.

47. Kiefer, J.; Wolfowitz, J. Stochastic Estimation of the Maximum of a Regression Function. Ann. Math. Stat. 1952, 23, 462-466. [CrossRef]

48. Virtanen, P.; Gommers, R.; Oliphant, T.E.; Haberland, M.; Reddy, T.; Cournapeau, D.; Burovski, E.; Peterson, P.; Weckesser, W.; Bright, J.; et al. SciPy 1.0: Fundamental algorithms for scientific computing in Python. Nat. Methods 2020, 17, 261-272. [CrossRef]

49. Whittaker, A.L.; Lymn, K.A.; Howarth, G.S. Effects of Metabolic Cage Housing on Rat Behavior and Performance in the Social Interaction Test. J. Appl. Anim. Welf. Sci. 2016, 19, 363-374. [CrossRef]

50. Sneddon, L.U.; Halsey, L.G.; Bury, N.R. Considering aspects of the 3Rs principles within experimental animal biology. J. Exp. Biol. 2017, 220, 3007-3016. [CrossRef]

51. Potter, D.; Jarrah, A.; Sakai, T.; Harrah, J.; Holliday, M.A. Character of function and size in kidney during normal growth of rats. Pediatr. Res. 1969, 3, 51-59. [CrossRef]

52. Filler, G.; Lee, M. Educational review: Measurement of GFR in special populations. Pediatr. Nephrol. 2018, 33, 2037-2046. [CrossRef] 